\title{
INVARIANT MEASURES OF STOCHASTIC PERTURBATIONS OF DYNAMICAL SYSTEMS USING FOURIER APPROXIMATIONS
}

\author{
MD SHAFIQUL ISLAM AND PAWEL GÓRA
}

\begin{abstract}
We consider dynamical system $\tau:[0,1] \rightarrow[0,1]$ and its stochastic perturbations $\bar{q}^{N}(\tau(x),),. N \geq 1$. Using Fourier approximation we construct a finite dimensional approximation $P_{N}$ to a perturbed Perron-Frobenius operator. Let $\hat{f}$ be an invariant density of $\tau$ and $f_{N}^{*}$ be a fixed point of $P_{N}$. We show that $\left\{f_{N}^{*}\right\}$ converge in $L^{1}$ to $\hat{f}$.
\end{abstract}

\section{IntRODUCTION}

Invariant measures of dynamical systems play important role in understanding the chaotic nature of dynamical systems. Let $(I, \mathcal{B}, \lambda)$ be a normalized measure space, where $I=[0,1], \mathcal{B}$ is a Borel $\sigma$-algebra of subsets of $I, \lambda$ Lebesgue measure in $(I, \mathcal{B})$. Let $\tau:(I, \mathcal{B}, \lambda) \rightarrow(I, \mathcal{B}, \lambda)$ be a deterministic dynamical system. The Frobenius-Perron operator $P_{\tau}$ of $\tau$ is a linear operator $P_{\tau}: L^{1}(I, \mathcal{B}) \rightarrow L^{1}(I, \mathcal{B})$ defined by

$$
\int_{A} P_{\tau} f(x) d \lambda(x)=\int_{\tau^{-1}(A)} f(x) d \lambda(x)
$$

for any $A \in \mathcal{B}$. It is well known [Boyarsky and Góra, 1997] that the fixed points of the Frobenius-Perron operator $P_{\tau}$ are the invariant densities of absolutely continuous invariant measures of $\tau$. Moreover, if $\tau$ is Markov with respect to a partition $\left\{I_{1}, I_{2}, \ldots \ldots, I_{q}\right\}$ of $I$, then the Frobenius-Perron operator $P_{\tau}$ is a finite dimensional matrix and it is relatively easier to study the absolutely continuous invariant measures of $\tau$ provided they exist [Boyarsky and Góra, 1997]. A non Markov dynamical system can be weakly approximated by Markov maps [Boyarsky and Góra, 1997, 2001; Billings and Bollt, 2001].

Physical systems are usually subjected to small perturbations from external noise or roundof errors. There are well-known results [Lasota and Mackey, 1994; Boyarsky and Góra, 1997] that study the stability of absolutely continuous invariant measures for measurable transformations. Consider the stochastically perturbed dynamical system $x \mapsto \tau(x)+\xi$ where $\xi$ is a additive noise which is applied once per each iteration. Let $\mathcal{P}(x, y)$ be the transition density of a transition from point $x$ to $y$ induced by noise $\xi$. In [Bollt et al., 2008] E. Bollt at. al. proposed a numerical method based on basis Markov partitions to

Date: February 13, 2010.

1991 Mathematics Subject Classification. 37A05, 37E05 .

Key words and phrases. Stochastic perturbations of dynamical systems; Fourier approximation; Frobenius-Perron operator; Probability density function; Absolutely continuous invariant measures;

This research is supported by the University of Prince Edward Island major research grants (MRG) of Shafiqul Islam and NSERC grant of Pawel Góra. 
approximate density functions of stochastically perturbed dynamical system $x \mapsto \tau(x)+\xi$. In this paper we consider Fourier approximation of $\xi$ and obtain a finite approximation of the Frobenius-Perron operator associated to the perturbed system. We present a convergence analysis of our method.

The paper is organized in the following way. In Section 2 invariant measures of stochastic perturbations of dynamical systems are discussed. In Section 3 we introduce a family of stochastic perturbation of dynamical systems and we show that the time evolution of densities of stochastic perturbations are given by linear operators. In Section 4 we present a matrix representation of operators in Section 3. We present stability and convergence analysis of our method in Section 5. Numerical examples are presented in Section 6.

\section{Stochastic PERTURbation AND InVARIANT MEASURE}

Let $L^{1}=L^{1}(I, \mathcal{B}, \lambda)$ and $\tau:[0,1] \rightarrow[0,1]$ be a piecewise monotonic mapping (see [Boyarsky and Góra, 1997]) on a partition $\mathcal{P}=\left\{0=b_{0}, b_{1}, \ldots, b_{q}=1\right\}$ and $P_{\tau}: L^{1} \rightarrow L^{1}$ be the Frobenius-Perron operator of $\tau$ defined in (1.1). For piecewise monotonic transformation $\tau$ the Frobenius-Perron operator $P_{\tau}$ has the following representation.

$$
P_{\tau} f(x)=\sum_{z \in\left\{\tau^{-1}(x)\right\}} \frac{f(z)}{\left|\tau^{\prime}(z)\right|} .
$$

Let $\bigvee(\cdot)$ be the standard one dimensional variation of a function and $B V(I)$ be the space of functions of bounded variations on $I$ equipped with the norm $\|\cdot\|_{B V}=\bigvee(\cdot)+\|\cdot\|_{L^{1}}$.

We consider Lasota-Yorke (see [Lasota and Yorke, 1973]) maps $\tau:[0,1] \rightarrow[0,1]$ such that $\left|\tau^{\prime}\right|>2$ and for every nonnegative density function $f \in B V([0,1])$ there exist constants $\beta>0$ and $0<\alpha<1$ such that

$$
\bigvee P_{\tau} f \leq \alpha \bigvee f+\beta\|f\|_{L^{1}}
$$

It was proved in [Lasota and Yorke, 1973] that Lasota-Yorke map $\tau$ satisfying 2.2 has an invariant density $\hat{f}$ of bounded variation and thus, an absolutely continuous invariant measure $\hat{\mu}=\hat{f} \cdot \lambda$.

For small $r>0$, let $w: \mathbb{R} \rightarrow \mathbb{R}^{+}$be a bounded function satisfying the following conditions:

(1) $w(t)=0$ for $|t|>r$,

(2) $w(-t)=w(t)$,

(3) $\int_{-r}^{r} w(t) d \lambda(t)=1$

It is easy to see that $w$ becomes Dirac's delta function as $r \rightarrow 0$. Let $q(x, y)$ be a kernel defined by

$$
q(x, y)=\left\{\begin{array}{ll}
w(y-x) & , x \in[r, 1-r) \\
w(y-x)+w(\bar{y}-x) & , x \in I-[r, 1-r]
\end{array},\right.
$$

where $\bar{y}=-y$ for $y \in[0, r)$ and $\bar{y}=1+(1-y)$ for $y \in(1-r, 1]$. The Markov process with transition density $p(x, \cdot)=q(\tau(x), \cdot)$ is called a stochastic perturbation of the map $\tau$. 
Let $Q: L^{1} \rightarrow L^{1}$ be the operator induced by the kernel $q(x, y)$ defined by

$$
(Q f)(y)=\int_{0}^{1} q(x, y) f(x) d \lambda(x) .
$$

It is proved by Góra in [Góra, 1984] that for any positive $f \in L^{1}$

$$
\bigvee(Q f) \leq 2 \bigvee f
$$

Treating $[0,1]$ as a circle and defining $q(x, y)=w(y-x)(\bmod 1)$, we show that the factor of 2 in the above inequality does not occur.

Lemma 2.1. For any $f \in L^{1}$ we have

$$
(Q f)(y)=(f * w)(y), y \in I
$$

where $g * h$ is the convolution of $g$ and $h$ defined by

$$
g * h(x)=\int g(y) h(x-y) d y=\int g(x-y) h(y) d y .
$$

Proof.

$$
(Q f)(y)=\int q(x, y) f(x) d \lambda(x)=\int w(x-y) f(x) d \lambda(x)=(f * w)(y)
$$

Lemma 2.2. For any positive $f \in L^{1}$ we have

$$
\bigvee(Q f) \leq \bigvee(f)
$$

Proof. For a fix integer $q \geq 1$ and a partition $0=t_{0}<t_{1}<\ldots<t_{q}=1$, we have

$$
\begin{aligned}
\sum_{i=1}^{q} \mid(Q f)\left(t_{i}\right) & -(Q f)\left(t_{i-1}\right)\left|=\sum_{i=1}^{q}\right|(f * w)\left(t_{i}\right)-(f * w)\left(t_{i-1}\right) \mid \\
& =\sum_{i=1}^{q}\left|(w * f)\left(t_{i}\right)-(w * f)\left(t_{i-1}\right)\right| \\
& =\sum_{i=1}^{q}\left|\int w(t) f\left(t_{i}-t\right) d t-\int w(t) f\left(t_{i-1}-t\right) d t\right| \\
& \leq \int\left(\sum_{i=1}^{q}\left|f\left(t_{i}-t\right)-f\left(t_{i-1}-t\right)\right|\right) w(t) d t \leq \int \bigvee(f) w(t) d t=\bigvee(f) .
\end{aligned}
$$


The time evolution under the densities of the stochastic perturbation $p(x, \cdot)=q(\tau(x), \cdot)$ of $\tau$ is given by

$$
\begin{aligned}
\left(P_{\mathrm{pert}} f\right)(y) & =\int_{I} p(x, y) f(x) d \lambda(x)=\int_{I} q(\tau(x), y) f(x) d \lambda(x) \\
& =\int_{I}\left(P_{\tau} f\right)(x) q(x, y) d \lambda(x)=\left(\left(Q \circ P_{\tau}\right) f\right)(y) .
\end{aligned}
$$

Thus,

$$
P_{\text {pert }}=Q \circ P_{\tau}
$$

and

$$
\bigvee P_{\text {pert }} f=\bigvee Q \circ P_{\tau} f \leq \bigvee P_{\tau} f \leq \alpha \bigvee f+\beta\|f\|_{L^{1}}
$$

Lemma 2.3. There is an $f^{*} \in L^{1}(0,1)$ of bounded variation such that $P_{\text {pert }} f^{*}=f^{*}$.

Proof. From inequality (2.7), $\left\{\bigvee P_{\text {pert }}^{n} f\right\}_{n \geq 1}$ is uniformly bounded in BV. By Helly's Theorem, $\left\{P_{\text {pert }}^{n} f\right\}$ is relatively compact, which implies by Kakutani-Yoshida Theorem, that

$$
\lim _{n \rightarrow \infty} \frac{1}{n} \sum_{i=0}^{n} P_{\text {pert }}^{i} f=f^{*} .
$$

for some $f^{*} \in L^{1}(0,1)$. It is easy to see that $f^{*}$ is a fixed point of $P_{\text {pert }}$ and that it is of bounded variation.

Theorem 2.4. Let $\tau:[0,1] \rightarrow[0,1]$ be a Lasota-Yorke (see [Lasota and Yorke, 1973]) map such that $\left|\tau^{\prime}\right|>2$ and for every nonnegative density function $f \in L^{1}([0,1])$ there exist constants $\beta>0$ and $0<\alpha<1$ such that $\bigvee_{0}^{1} P_{\tau} f \leq \alpha \bigvee f+\beta\|f\|_{L^{1}}$. If the above kernel $q(x, y)$ satisfies (2.3), then the stochastic perturbation $p(x,)=.q(\tau(x),$.$) of the map \tau$ has an invariant density $f^{*}$.

Proof. The proof follows from Lemma 2.1, Lemma 2.2 and Lemma 2.3.

In the following section we consider a family $q^{N}(\cdot, \cdot), N \geq 1$ of doubly stochastic kernels and corresponding stochastic perturbations $p^{N}(x, \cdot)=q^{N}(\tau(x), \cdot), N \geq 1$ of Lasota-Yorke map $\tau:[0,1] \rightarrow[0,1]$. They will be constructed in such a way that the corresponding operator $P_{\text {pert }}$ are finite dimensional. We will prove the existence of invariant probability measures $\mu_{N}$ of the stochastic perturbations $p^{N}(x, \cdot)=q^{N}(\tau(x), \cdot)$ of the map $\tau$. Our main objective is to show that the limit points (limit measures) $\mu$ of the set $\left\{\mu_{N}: N \geq 1\right\}$ are of the form $\mu=\hat{f} \cdot \lambda$, where $\hat{f}$ is the invariant density of $\tau$. 


\section{FAmily of STOCHASTIC PERTURBATIONS AND INVARIANT MEASURES}

Now, we define a family of probability densities $\bar{q}^{N}(x, y), N=1,2, \ldots$ as follows: let $\left\{g_{N}\right\}_{N \geq 1}$ be a sequence of $C^{2}$ nonnegative functions with support in $[-1 / 2,1 / 2]$ such that $g_{N}$ is symmetric with respect to $y$ axis, $g_{N}(-1 / 2)=g_{N}(1 / 2)$ for all $N \geq 1$ and which converges to Dirac's delta function as $N \rightarrow \infty$. Each $g_{N}$, which can be also seen as a 1 -periodic on the whole real line, can be approximated by its partial Fourier sum arbitrary close in the supremum norm. Let

$$
h_{N}(\xi)=c_{S}+a_{0, N}+2 \sum_{s=1}^{S}\left(a_{s, N} \cos (2 s \pi \xi)+b_{s, N} \sin (2 s \pi \xi)\right)
$$

where $S$ can be chosen independently of $N$, be an approximation obtained from Fourier approximation by shifting it up by a small constant $c_{S}$ to ensure $h_{N} \geq 0$ on $[-1 / 2,1 / 2]$. We have $c_{S} \rightarrow 0$ as $S \rightarrow \infty$. We can also make $h_{N}$ converge to Dirac's delta $\delta_{0}$ as $N \rightarrow \infty$. Let $L=\int_{-1 / 2}^{1 / 2} h_{N}(t) d t$. Define a family of functions $w^{N}$ :

$$
w^{N}(t)=\frac{1}{L} h_{N}(t), N=1,2,3, \ldots
$$

Now we define a family of probability densities $q^{N}(x, y), N=1,2, \ldots$ as follows:

$$
q^{N}(x, y)=w^{N}(x-y), N=1,2,3, \ldots
$$

Thus,

$$
\begin{aligned}
q^{N}(x, y)= & w^{N}(x-y) \\
= & \frac{1}{L}\left[c_{S}+a_{0, N}+2 \sum_{s=1}^{S}\left(a_{s, N} \cos (2 s \pi(x-y))+b_{s, N} \sin (2 s \pi(x-y))\right)\right] \\
= & \frac{1}{L}\left[c_{S}+a_{0, N}+2 \sum_{s=1}^{S}\left(a_{s, N}(\cos (2 s \pi x) \cos (2 s \pi y)+\sin (2 s \pi x) \sin (2 s \pi y))\right.\right. \\
& \left.\left.\quad+b_{s, N}(\sin (2 s \pi x) \cos (2 s \pi y)-\cos (2 s \pi x) \sin (2 s \pi y))\right)\right] .
\end{aligned}
$$

The family of transition densities $p^{N}(x, \cdot)=q^{N}(\tau(x), \cdot)$ induces a family of stochastic perturbation of the map $\tau$. For $N=1,2, \ldots$ let $Q_{N}: L^{1} \rightarrow L^{1}$ be the operator induced by the kernel $q^{N}(x, y)$ defined by

$$
\left(Q_{N} f\right)(y)=\int_{0}^{1} q^{N}(x, y) f(x) d \lambda(x)
$$


The time evolution of the densities of the stochastic perturbation $p^{N}(x, \cdot)=q^{N}(\tau(x), \cdot)$ of $\tau$ is given by

$$
\begin{aligned}
\left(P_{N} f\right)(y) & =\int_{I} p^{N}(x, y) f(x) d \lambda(x)=\int_{I} q^{N}(\tau(x), y) f(x) d \lambda(x) \\
& =\int_{I}\left(P_{\tau} f\right)(x) q^{N}(x, y) d \lambda(x)=\left(\left(Q_{N} \circ P_{\tau}\right) f\right)(y) .
\end{aligned}
$$

Thus,

$$
P_{N}=Q_{N} \circ P_{\tau}
$$

From Section 2 we have

$$
\bigvee_{0}^{1} P_{N} f=\bigvee_{0}^{1} Q_{N} \circ P_{\tau} f \leq \bigvee_{0}^{1} P_{\tau} f \leq \alpha \bigvee f+\beta\|f\|_{L^{1}}
$$

Thus, by Theorem 2.4, for each $N \geq 1$, the operator $P_{N}$ has a fixed point $f_{N}^{*}$.

\section{Matrix Representation of $P_{N}$}

Let us define:

$$
\begin{aligned}
u_{0}(x) & =1 ; \\
u_{4 s+1}(x) & =\cos (2(s+1) \pi x), s=0,1,2, \ldots S-1 ; \\
u_{4 s+2}(x) & =\sin (2(s+1) \pi x), s=0,1,2, \ldots S-1 ; \\
u_{4 s+3}(x) & =\sin (2(s+1) \pi x), s=0,1,2, \ldots S-1 ; \\
u_{4 s+4}(x) & =\cos (2(s+1) \pi x), s=0,1,2, \ldots S-1 ; \\
v_{0}(x) & =1 ; \\
v_{4 s+1}(x) & =\cos (2(s+1) \pi x), s=0,1,2, \ldots S-1 ; \\
v_{4 s+2}(x) & =\sin (2(s+1) \pi x), s=0,1,2, \ldots S-1 ; \\
v_{4 s+3}(x) & =\cos (2(s+1) \pi x), s=0,1,2, \ldots S-1 ; \\
v_{4 s+4}(x) & =\sin (2(s+1) \pi x), s=0,1,2, \ldots S-1 ;
\end{aligned}
$$

Let $K=4 S$ and let the matrix $A=\left(A_{m n}\right)_{0 \leq m, n \leq K}$ be the diagonal matrix with the diagonal

$$
\begin{array}{r}
\frac{1}{L}\left(c_{S}+a_{0, N}, 2 a_{1, N}, 2 a_{1, N}, 2 b_{1, N},-2 b_{1, N}, 2 a_{2, N}, 2 a_{2, N}, 2 b_{2, N},-2 b_{2, N},\right. \\
\left.\ldots, 2 a_{S, N}, 2 a_{S, N}, 2 b_{S, N},-2 b_{S, N}\right) .
\end{array}
$$

Thus, $q^{N}(x, y)=\sum_{m, n=0}^{K} A_{m n} u_{n}(x) v_{m}(y)$,

The kernel $q^{N}(\cdot, \cdot)$ defined above satisfies the following properties:

(1) $q^{N}(x, y) \geq 0$.

(2) $q^{N}(\cdot, \cdot)$ is measurable as functions of two variables,

(3) For every $x \in I$ we have $\int_{I} q^{N}(x, y) d y=1$, 
(4) For every $y \in I$ we have $\int_{I} q^{N}(x, y) d x=1$,

(5) $q^{N}(x, y) \equiv q^{N}(x \bmod 1, \mathrm{y} \bmod 1)$,

(6) $q^{N}(x, y)=\sum_{m, n=0}^{K} A_{m n} u_{n}(x) v_{m}(y)$,

(7) Let $B(x, r)=\{y:|x-y|<r\}$ and $c_{N}(x, r)=\int_{I \backslash B(x, r)} q_{n}(x, y) d y$. Then for any $r>0$,

$$
c_{N}(r)=\sup _{x \in I} c_{N}(x, r) \rightarrow 0
$$

as $N \rightarrow+\infty$.

We have

$$
\begin{aligned}
{\left[P_{N} f\right](y) } & =\int_{0}^{1} \sum_{m, n=0}^{K} A_{m n} u_{n}(\tau(x)) v_{m}(y) f(x) d x \\
& =\sum_{m, n=0}^{K} A_{m n}\left[\int_{0}^{1} u_{n}(\tau(x)) f(x) d x\right] v_{m}(y) \\
& =\sum_{m, n=0}^{K}\left[\int_{0}^{1} u_{n}(\tau(x)) f(x)\right] \bar{v}_{m}(y)
\end{aligned}
$$

for $y \in I$, where,

$$
\bar{v}_{n}(y)=\sum_{m=0}^{K} A_{m n} v_{m}(y), n=0,1,2, \ldots K .
$$

Thus, any initial density $f$ is projected by the operator $P_{N}$ into the vector space $\Delta_{N}$ spanned by the functions $\overline{v_{n}}, n=0, \ldots, K$, that is,

$$
\left(P_{N} f\right)(y)=\sum_{n=0}^{K} q_{n}^{\prime} \bar{v}_{n}(x)
$$

where

$$
q_{n}^{\prime}=\int_{0}^{1} u_{n}(\tau(x)) f(x) d x .
$$

We are interested in finding the matrix representation of the operator $P_{N}$.

Assuming that a given density $f(x)$ belongs to the space $\Delta_{N}$, we can expand it in the basis,

$$
f(x)=\sum_{m=0}^{K} q_{m} \bar{v}_{m}(x) .
$$

Let $B$ denote a matrix of integrals,

$$
B_{n m}=\int_{0}^{1} u_{n}(\tau(x)) v_{m}(x) d x,
$$

where $n, m=0, \ldots, K$. Observe that $B$ depends directly on the system $\tau$ and on the noise via the basis functions $u$ and $v$. Let us define

$$
D=B A .
$$


Lemma 4.1. The matrix $D$ in (4.5) is the representation of the operator $P_{N}$ with respect to the basis $\left\{\bar{v}_{l}\right\}_{l=0}^{K}$.

Proof. All we need to show is the following: $q_{n}^{\prime}=\sum_{m=0}^{K} D_{n m} q_{m}, \quad n=0,1,2, \ldots K$. Now,

$$
\begin{aligned}
\sum_{m=0}^{K} D_{n m} q_{m}= & D_{n 0} q_{0}+D_{n 1} q_{1}+\ldots D_{n K} q_{K} \\
= & \left(\sum_{l=0}^{K} B_{n l} A_{l 0}\right) q_{0}+\left(\sum_{l=0}^{K} B_{n l} A_{l 1}\right) q_{1}+\ldots+\left(\sum_{l=0}^{K} B_{n l} A_{l K}\right) q_{K} \\
= & \left(\sum_{l=0}^{K}\left\{\int_{0}^{1} u_{n}(\tau(x)) v_{l}(x) d x\right\} A_{l 0}\right) q_{0}+\left(\sum_{l=0}^{K}\left\{\int_{0}^{1} u_{n}(\tau(x)) v_{l}(x) d x\right\} A_{l 1}\right) q_{1} \\
& +\ldots+\left(\sum_{l=0}^{K}\left\{\int_{0}^{1} u_{n}(\tau(x)) v_{l}(x) d x\right\} A_{l K}\right) q_{K} .
\end{aligned}
$$

On the other hand

$$
\begin{aligned}
q_{n}^{\prime} & =\int_{0}^{1} u_{n}(\tau(x)) f(x) d x=\int_{0}^{1} u_{n}(\tau(x))\left(\sum_{m=0}^{K} q_{m} \bar{v}_{m}(x)\right) d x \\
& =\int_{0}^{1} u_{n}(\tau(x))\left(\sum_{m=0}^{K} q_{m}\left(\sum_{l=0}^{K} A_{l m} v_{l}(x)\right)\right) d x \\
& =\int_{0}^{1} u_{n}(\tau(x))\left[\left(\sum_{l=0}^{K} A_{l 0} v_{l}(x)\right) q_{0}+\left(\sum_{l=0}^{K} A_{l 1} v_{l}(x)\right) q_{1}+\ldots+\left(\sum_{l=0}^{K} A_{l K} v_{l}(x)\right) q_{K}\right] d x \\
& =\left(\sum_{l=0}^{K}\left\{\int_{0}^{1} u_{n}(\tau(x)) v_{l}(x) d x\right\} A_{l 0}\right) q_{0}+\left(\sum_{l=0}^{K}\left\{\int_{0}^{1} u_{n}(\tau(x)) v_{l}(x) d x\right\} A_{l 1}\right) q_{1}+\ldots \\
& =\sum_{m=0}^{K} D_{n m} q_{m} .
\end{aligned}
$$

In this way we have arrived at a representation of the operator $P_{N} f$ by a matrix $D$ of size $(K+1) \times(K+1)$ with respect to the basis $\left\{\bar{v}_{k}\right\}_{k=0}^{K}$, the elements of which read,

$$
D_{n m}=\int_{0}^{1} u_{n}(\tau(x)) \overline{v_{m}}(x) d x, \quad n, m=0, \ldots, K .
$$


Recall from Section 3

$$
\left(Q_{N} f\right)(y)=\int_{0}^{1} q^{N}(x, y) f(x) d \lambda(x)
$$

Lemma 5.1. For any $f \in L^{1}$ we have $Q_{N} f \rightarrow f$ as $N \rightarrow \infty$ in the $L^{1}$ norm. The convergence is uniform on relatively compact subsets of $L^{1}$.

Proof. It can be shown that for each $N \geq 1,\left\|Q_{N}\right\|_{1}=1$. Let $f \in L^{1}$ and $\epsilon>0$. Since continuous functions are dense in $L^{1}$, there exists a continuous function $g$ in $I$ such that $\|g-f\|_{1}<\frac{\epsilon}{3}$. Since $g$ is continuous it is uniformly continuous in $[0,1]$. Thus,

$$
\left\|Q_{N} f-f\right\|_{1} \leq\left\|Q_{N} f-Q_{N} g\right\|_{1}+\left\|Q_{N} g-g\right\|_{1}+\|g-f\|_{1}
$$

Now,

$$
\begin{aligned}
\left\|Q_{N} g-g\right\|_{1} & =\int\left|g(y)-\left(Q_{N} g\right)(y)\right| d y=\int\left|g(y)-\int g(x) q^{N}(x, y) d x\right| d y \\
& \leq \iint|g(y)-g(x)| q^{N}(x, y) d x d y \leq \frac{\epsilon}{3} \iint q^{N}(x, y) d x d y=\frac{\epsilon}{3} .
\end{aligned}
$$

This proves

$$
\left\|Q_{N} f-f\right\|_{1} \leq \epsilon
$$

Lemma 5.2. Let $f_{N} \in \Delta_{N}$ and $f_{N}=\sum_{j=0}^{N} c_{j} \bar{v}_{j}(x)$. Then $P_{N} f_{N}=f_{N}$ if and only if $D c=c$ where $c$ is the transpose of $\left(c_{0}, c_{1}, \ldots, c_{N}\right)$.

Proof. Let $f_{N}=\sum_{j=0}^{N} c_{j} \bar{v}_{j}(x)$. Then

$$
\begin{aligned}
P_{N} f_{N} & =P_{N}\left(\sum_{j=0}^{N} c_{j} \bar{v}_{j}(x)\right)=\sum_{j=0}^{N} c_{j} P_{N} \bar{v}_{j}(x) \\
& =c_{0}\left(\sum_{i=0}^{N} D_{i 0} \bar{v}_{i}(x)\right)+c_{1}\left(\sum_{i=0}^{N} D_{i 1} \bar{v}_{i}(x)\right)+\ldots+c_{N}\left(\sum_{i=0}^{N} D_{i N} \bar{v}_{i}(x)\right) \\
& =\left(\sum_{l=0}^{N} D_{0 l} c_{l}\right) \bar{v}_{0}(x)+\left(\sum_{l=0}^{N} D_{1 l} c_{l}\right) \bar{v}_{1}(x)+\ldots+\left(\sum_{l=0}^{N} D_{N l} c_{l}\right) \bar{v}_{N}(x) .
\end{aligned}
$$


Thus, $P_{N} f_{N}=f_{N}$ if and only if

$$
\begin{gathered}
\sum_{l=0}^{N} D_{0 l} c_{l}=c_{0} \\
\sum_{l=0}^{N} D_{1 l} c_{l}=c_{1} \\
\vdots \\
\sum_{l=0}^{N} D_{K l} c_{l}=c_{N}
\end{gathered}
$$

That is,

$$
D c=c .
$$

Now we prove the main theorem of this section.

Theorem 5.3. Let $\tau:[0,1] \rightarrow[0,1]$ be a Lasota-Yorke (see [Lasota and Yorke, 1973]) map such that $\left|\tau^{\prime}\right|>2$ and for every nonnegative density function $f \in L^{1}([0,1])$ there exist constants $\beta>0$ and $0<\alpha<1$ such that $\bigvee_{0}^{1} P_{\tau} f \leq \alpha \bigvee f+\beta\|f\|_{L^{1}}$. Let $f_{N}^{*} \in \Delta_{N}$ be an invariant density of stochastic perturbation $q^{N}(\tau(x), \cdot)$ of $\tau$ such that $P_{N} f_{N}^{*}=f_{N}^{*}$. Then the set $\left\{f_{N}^{*}\right\}_{N \geq 1}$ is relatively compact in $L^{1}$ and any limit point of $\left\{f_{N}^{*}\right\}_{N \geq 1}$ is a $\tau$ invariant density $\hat{f}$.

Proof. By inequality (2.7),

$$
\bigvee f_{N}^{*}=\bigvee\left(P_{N} f_{N}^{*}\right)=\bigvee\left(\left(Q_{N} \circ P_{\tau}\right) f_{N}^{*} \leq \bigvee\left(P_{\tau} f_{N}^{*}\right) f_{N}^{*}\right) \leq \alpha \bigvee f+\beta\|f\|_{L^{1}}
$$

Thus, the set $\left\{f_{N}^{*}\right\}_{N \geq 1}$ is uniformly bounded in variation. By Helly's Theorem, $\left\{f_{N}^{*}\right\}_{N \geq 1}$ is relatively compact in $L^{1}$. Let $f_{N_{i}^{*}}$ be a subsequence of $f_{N}$ and $f_{N_{i}^{*}} \rightarrow f$ in $L^{1}$. Then,

$$
\begin{aligned}
\left\|\hat{f}-P_{\tau} \hat{f}\right\|_{1} \leq & \left\|\hat{f}-f_{N_{i}}^{*}\right\|_{1}+\left\|f_{N_{i}}^{*}-Q_{N_{i}} P_{\tau} f_{N_{i}}^{*}\right\|_{1} \\
& +\left\|Q_{N_{i}} P_{\tau} f_{N_{i}}^{*}-Q_{N_{i}} P_{\tau} \hat{f}\right\|_{1}+\left\|Q_{N_{i}} P_{\tau} \hat{f}-P_{\tau} \hat{f}\right\|_{1} .
\end{aligned}
$$

Using Lemma 5.1 and the definition of $P_{N}$ it is easy to see that $P_{\tau} \hat{f}=\hat{f}$.

\section{EXAMPLES}

Our approximation method uses as "building blocks" trigonometric functions which have the same values at 0 and at 1 . Therefore, the method is not best suited to approximate densities which do not have this property. To go around this deficiency we use a symmetrization of the map.

Example 6.1. For $0<\alpha<1,0<p \leq 1, q>0$, consider the deterministic dynamical system $\tau_{1}:[0,1] \rightarrow[0,1]$ defined by 


$$
\tau_{1}(x)= \begin{cases}\frac{\alpha x}{\alpha p+(\alpha-p) x} & , x \in[0, \alpha] \\ \frac{q(1-\alpha)(1-x)}{q-q \alpha-\alpha+(1-q+q \alpha) x} & , x \in(\alpha, 1]\end{cases}
$$

We set

$$
\alpha=\frac{1}{2}, p=\frac{1}{3}, q=6 .
$$

It can be shown that $\tau_{1}$-invariant probability density is

$$
f_{1}(x)=\frac{1+\beta}{\beta^{2}\left(x+\frac{1}{\beta}\right)^{2}},
$$

where for our values of constants $\beta=-\frac{1}{2}$ [Schweiger, 1983]. Let $\tau_{2}:[0,1] \rightarrow[0,1]$ defined by $\tau_{2}(x)=1-\tau_{1}(1-x)$. $\tau_{2}$ is conjugated to $\tau_{1}$ by homeomorphism $h(x)=1-x$. It can be easily proved that $\tau_{2}$-invariant density is $f_{2}(x)=f_{1}(1-x)$, where $f_{1}$ is $\tau_{1}$-invariant. Let $\tau:[0,1] \rightarrow[0,1]$ be defined by

$$
\tau(x)= \begin{cases}\frac{1}{2} \tau_{1}(2 x) & , x \in\left[0, \frac{1}{2}\right] \\ \frac{1}{2}+\frac{1}{2} \tau_{2}\left(2\left(x-\frac{1}{2}\right)\right) & , x \in\left(\frac{1}{2}, 1\right]\end{cases}
$$

$\tau$-invariant density is

$$
f_{\tau}(x)= \begin{cases}f_{1}(2 x) & , \text { for } 0 \leq x \leq \frac{1}{2} \\ f_{2}\left(2\left(x-\frac{1}{2}\right)\right) & , \text { for } \frac{1}{2}<x \leq 1\end{cases}
$$

which is symmetric with respect $x=1 / 2$ so $f_{\tau}(0)=f_{\tau}(1)$.

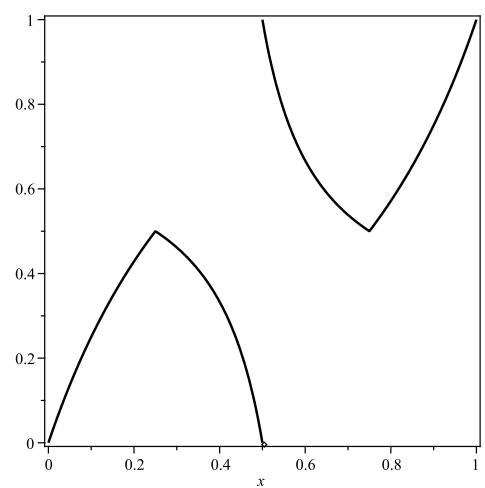

Figure 1. The transformation $\tau$

Consider the stochastic perturbation of the above deterministic dynamical systems $\tau$ by the noise $g_{N}(\xi)=N g(N \xi), g(\xi)=e^{-\xi^{2}}$ restricted to $[-1 / 2,1 / 2]$ and extended periodically to whole real line, $N \geq 1$. In particular, we consider the dynamical systems $\tau$ with $\alpha=$ $\frac{1}{2}, p=\frac{1}{3}, q=6$ and the noise $g_{N}$ with $N=15$. The Fourier approximation of $g_{15}$, with $S(15)=10$ is 


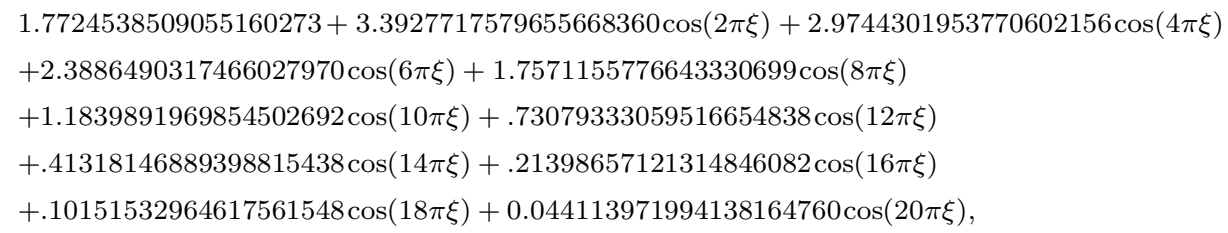

where we have chosen $C_{S(15)}=0.0320895553170388570$ to ensure that the Fourier approximation is positive on $[-1 / 2,1 / 2]$. After normalization we obtain
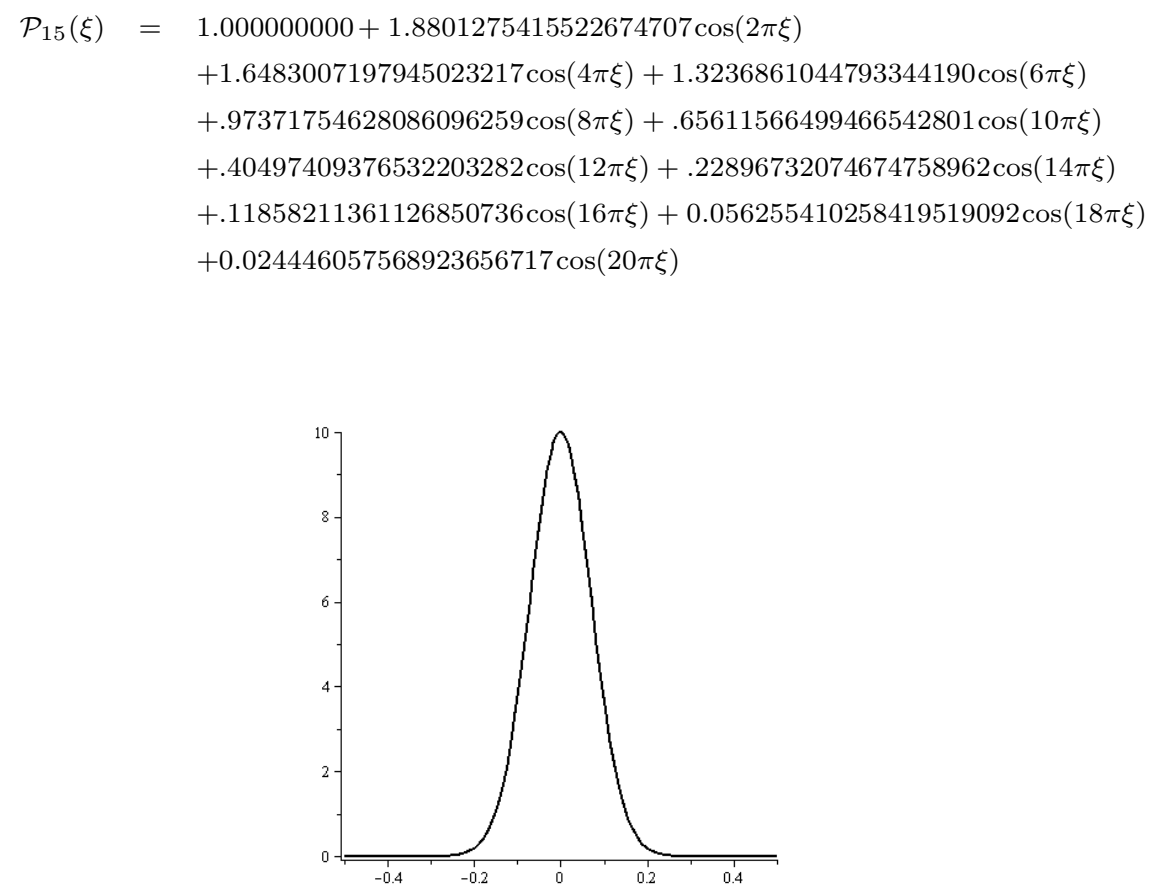

Figure 2. The transition density $P_{15}$

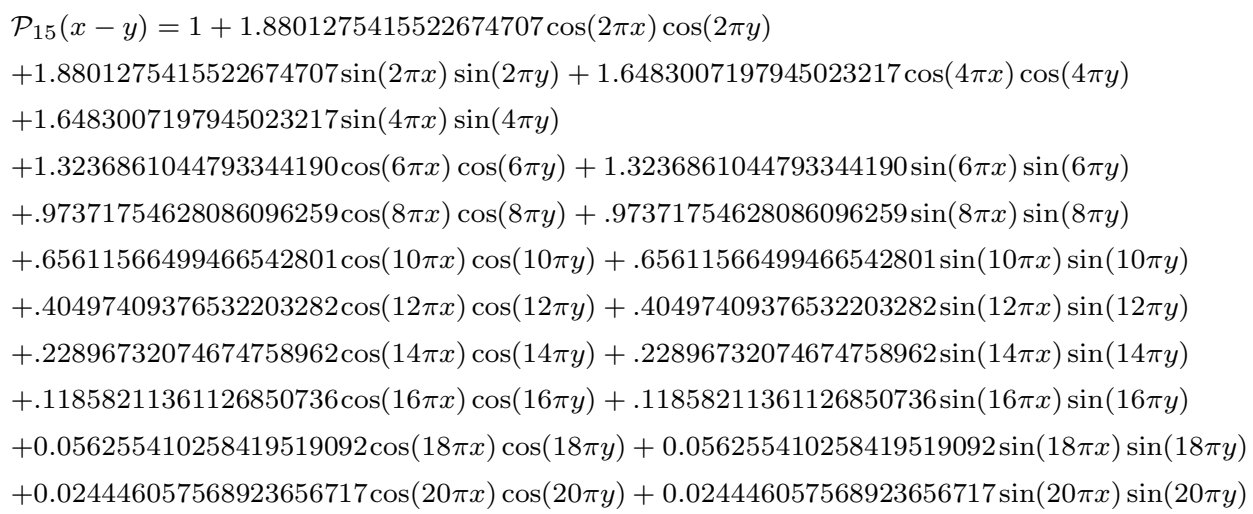


From equation 4.1 we obtain $u$ 's and $v$ 's for $s=0,1, \ldots, 20$. Then, the matrix $A=$ $\left(A_{m n}\right)_{0 \leq m, n \leq 20}$, is the diagonal matrix with diagonal

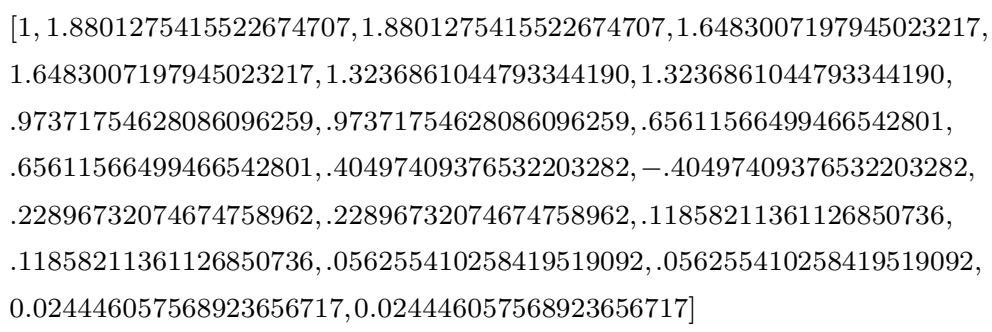

and we have

$$
\bar{v}_{m}=A_{m m} v_{m}, m=0,1,2, \ldots 20 .
$$

For the above perturbed dynamical system we compute the matrix $D$ in (4.5). The eigenvector of the matrix $D$ for the eigenvalue 1 is :

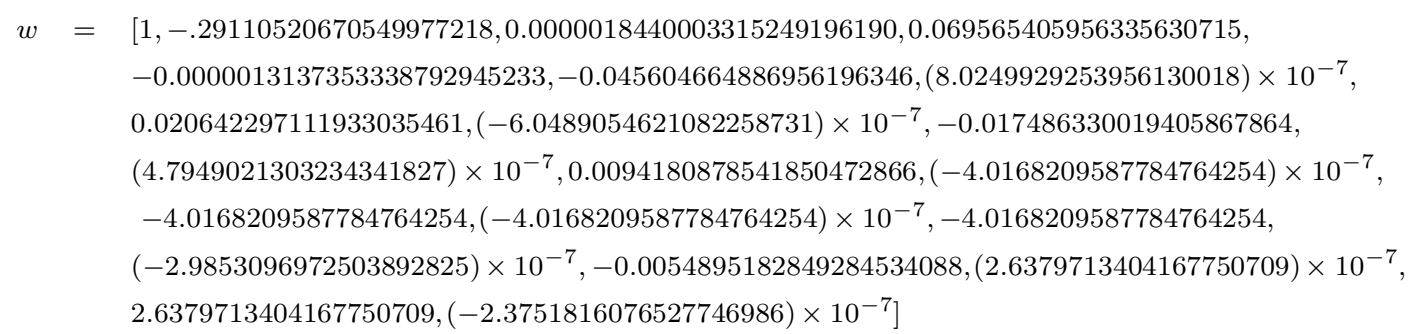

and it provides an approximation $f_{15}^{*}=\sum_{0}^{20} w_{m} \bar{v}_{m}$ to the $\tau$-invariant density (Fig. 3 ) $\hat{f}$. Much better approximations shown in Fig. 4 and Fig. 5 are obtained by taking $N=20,30$ and $S=15,20$ respectively, which results in matrix $D$ of size $2 S+1=31,41$ respectively.

Errors in $L^{1}-$ norms are listed in the following table.

\begin{tabular}{|c|c|c|}
\hline$N$ & $S$ & $\left\|f_{N}^{*}-\hat{f}\right\|$ \\
\hline 15 & 10 & 0.025044041879482 \\
\hline 20 & 15 & 0.018413171411567 \\
\hline 30 & 20 & 0.011280614132958 \\
\hline
\end{tabular}




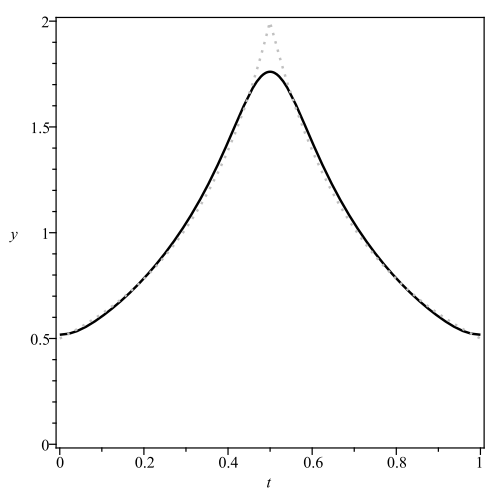

FiguRE 3. An approximation $f_{15}^{*}$ to the invariant density $\hat{f}$ of the map $\tau$ obtained as an invariant density of transition matrix $D$ of size $21 \times 21$

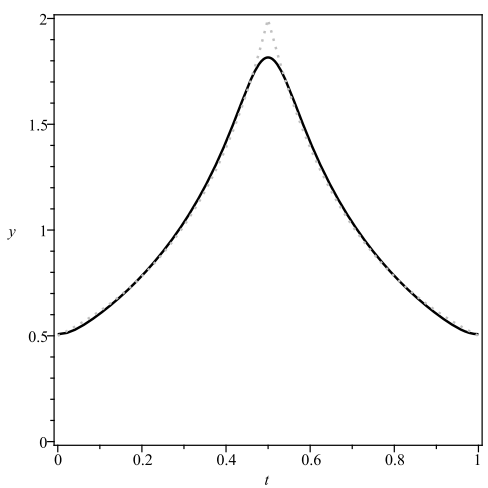

Figure 4. An approximation $f_{30}^{*}$ to the invariant density $\hat{f}$ of the map $\tau$ obtained as an invariant density of transition matrix $D$ of size $31 \times 31$ 


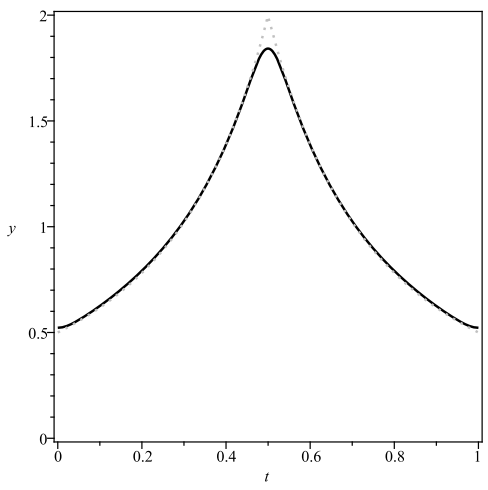

Figure 5. An approximation $f_{30}^{*}$ to the invariant density $\hat{f}$ of the map $\tau$ obtained as an invariant density of transition matrix $D$ of size $41 \times 41$

\section{REFERENCES}

Billings, L., Bollt, E. M. [2001], Probability density functions of some skew tent maps, Chaos Solitons Fractals 12, no. 2, 365-376.

Bollt, E., Góra, P., Ostruszka, A., Zyczkowski, K. [2008], Basis Markov partitions and transition matrices for stochastic systems, SIAM J. Appl. Dyn. Syst. 7, no. 2, 341-360.

Boyarsky, A. and Góra, P. [1997], Laws of Chaos, Birkhaüser.

Boyarsky, A. and Góra, P. [2001], Invariant measures for Chebyshev maps, J. Appl. Math. Stochastic Anal. 14, no. 3, 257-264.

Góra, P. [1984], On small stochastic perturbations of mappings of the unit intervals, Colloquium Mathematicum XLIX, no. 3, 73-85.

Lasota, A., Mackey, M. C. [1994], Chaos, fractals, and noise. Stochastic aspects of dynamics, Applied Mathematical Sciences, 97. Springer-Verlag, New York.

Lasota, A. and Yorke, J.A. [1973], On the existence of invariant measures for piecewise monotonic transformations, Trans. Amer. Math. Soc. 186,481-488.

Schweiger, F. [1983], Invariant measures for piecewise linear fractional maps, J. Austral. Math. Soc. 34, 55-59.

Department of Mathematics and Statistics, University of Prince Edward Island, 550 University Ave, Charlottetown, PE, C1A 4P3, Canada.

E-mail address: sislam@upei.ca

Department of Mathematics and Statistics, Concordia University, Montreal, Quebec, CANADA.

E-mail address: pgora@mathstat. concordia.ca 